\title{
Assessment of clinical effect and treatment quality of immediate-release carvedilol-IR versus SLOW release carvedilol-SR in Heart Failure patients (SLOW-HF): study protocol for a randomized controlled trial
}

Dong-Ju Choi ${ }^{1 \dagger}$, Chan Soon Park ${ }^{1 \dagger}$, Jin Joo Park ${ }^{1,14^{*}}$ (D, Hae-Young Lee ${ }^{2}$, Seok-Min Kang ${ }^{3}$, Byung-Su Yoo ${ }^{4}$, Eun-Seok Jeon ${ }^{5}$, Seok Keun Hong ${ }^{6}$, Joon-Han Shin ${ }^{7}$, Myung-A Kim ${ }^{8}$, Dae-Gyun Park ${ }^{9}$, Eung-Ju Kim ${ }^{10}$, Soon-Jun Hong ${ }^{11}$, Seok Yeon Kim ${ }^{12}$ and Jae-Joong Kim ${ }^{13}$

\begin{abstract}
Background: Carvedilol is a non-selective, third-generation beta-blocker and is one of the cornerstones for treatment for patients with heart failure and reduced ejection fraction (HFrEF). However, due to its short half-life, immediate-release carvedilol (IR) needs to be prescribed twice a day. Recently, slow-release carvedilol (SR) has been developed. The aim of this study is to evaluate whether carvedilol-SR is non-inferior to standard carvedilol-IR in terms of its clinical efficacy in patients with HFrEF.

Methods/design: Patients with stable HFrEF will be randomly assigned in a 1:1 ratio to the carvedilol-SR group (160 patients) and the carvedilol-IR group (160 patients). Patients aged $\geq 20$ years, with a left ventricular ejection fraction $\leq 40 \%$, N-terminal pro B-natriuretic peptide (NT-proBNP) $\geq 125 \mathrm{pg} / \mathrm{ml}$ or BNP $\geq 35 \mathrm{pg} / \mathrm{ml}$, who are clinically stable and have no evidence of congestion or volume retention, will be eligible. After randomization, patients will be followed up for 6 months. The primary endpoint is the change in NT-proBNP level from baseline to the study end. The secondary endpoints include the proportion of patients with NT-proBNP increment $>10 \%$ from baseline, composite of all-cause mortality and readmission, mortality rate, readmission rate, changes in blood pressure, quality of life, and drug compliance.
\end{abstract}

Discussions: The SLOW-HF trial is a prospective, randomized, open-label, phase-IV, multicenter study to evaluate the therapeutic efficacy of carvedilol-SR compared to carvedilol-IR in HFrEF patients. If carvedilol-SR proves to be non-inferior to carvedilol-IR, a once-daily prescription of carvedilol may be recommended for patients with HFrEF.

Trial registration: ClinicalTrials.gov, ID: NCT03209180. Registered on 6 July 2017.

Keywords: Heart failure with reduced ejection fraction, Carvedilol, Slow release, Immediate release, Clinical efficacy, NT-proBNP

\footnotetext{
* Correspondence: jinjooparkmd@gmail.com

${ }^{\dagger}$ Equal contributors

'Department of Internal Medicine, Seoul National University College of Medicine, Seoul National University Bundang Hospital, Seongnam, South Korea

${ }^{14}$ Cardiovascular Center and Division of Cardiology, Department of Internal Medicine, Seoul National University Bundang Hospital, 82 Gumi-ro 173

Beon-gil, Bundang-gu, Gyeonggi-do 13620, South Korea

Full list of author information is available at the end of the article
} 


\section{Background}

Heart failure (HF) is a clinical syndrome characterized by typical symptoms and signs that result from abnormal cardiac structure or function [1]. It is globally present with an increasing prevalence and is associated with high morbidity and mortality $[2,3]$. The current treatment of HF targets includes the prevention of acute decompensation and improving the long-term survival of HF patients [4].

Neuro-humoral activation plays a crucial role in development and progression of HF [5]. Beta-blockers reduce the increased sympathetic tone in HF patients and have been shown to improve the survival in patients with HF and a reduced ejection fraction (HFrEF) [6-8]. Carvedilol is a non-selective beta-blocker which has been most extensively studied in HF patients with mild-to-severe left ventricular (LV) dysfunction [8,9]. Carvedilol has also shown additional mortality risk reduction in HFrEF patients compared to metoprolol [10].

Current HF guidelines recommend the administration of carvedilol twice daily $[1,11]$. This is due to rapid absorption and metabolism of carvedilol whose plasma concentration reaches a peak within 1-2 $\mathrm{h}$ after administration and shows an elimination half-life of 7-10 h after peak concentration [12]. Although the drug compliance of carvedilol was comparable between once- and twice-daily dosing in one report [13]; however, there has been a concern that regimen complexity is inversely associated with medication adherence and related to even worse clinical outcomes in other studies and real-world clinical practice [14-16].

In the "Assessment of clinical effect and treatment quality of immediate-release carvedilol-IR versus SLOW-release carvedilol-SR in Heart Failure patients (SLOW-HF): a prospective, randomized, open-label, multicenter study," we aim to evaluate the clinical efficacies of immediate-release carvedilol (IR) and slowrelease carvedilol (SR) forms of carvedilol in Asian HFrEF patients.

\section{Methods/design \\ Overview}

The study flow is presented in Fig. 1. This trial is a prospective, randomized, open-label, phase-IV, multicenter trial to evaluate the efficacy of carvedilol-SR. The aim of this study is to evaluate whether carvedilol-SR is noninferior to standard carvedilol-IR in terms of its clinical efficacy in patients with HFrEF. The protocol of this trial has been registered at ClinicalTrials.gov (registration number: NCT03209180). The protocol follows the Standard Protocol Items: Recommendations for Interventional Trials (SPIRIT) Checklist (Additional file 1).

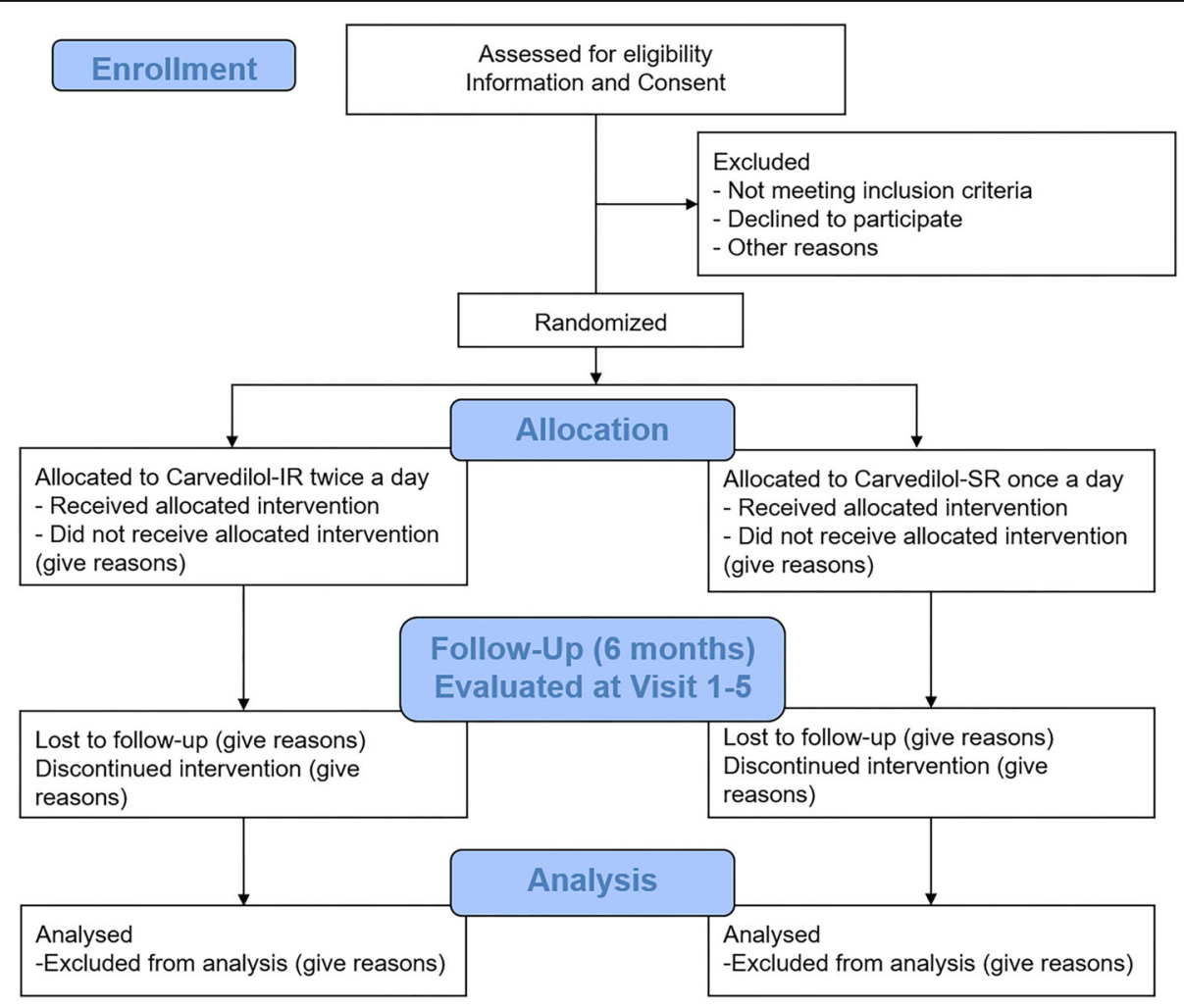

Fig. 1 Study flow chart. BNP B-type natriuretic peptide, HFrEF heart failure with reduced ejection fraction, IR immediate-release, LVEF left ventricular ejection fraction, NT-proBNP N-terminal B-type natriuretic peptide, SR slow-release 


\section{Study population}

Patients who are at aged 20 years or older and with a confirmed diagnosis of HFrEF (left ventricular ejection fraction $(\mathrm{LVEF}) \leq 40 \%)$ within the pre-analytical 6 months will be included in this study. Transthoracic echocardiography was operated at each institute according to the recommendations of the American Society of Echocardiography using commercially available systems [17]. In addition, the patients should be clinically stable without evidence of congestion and fluid retention, and have an increased level of $\mathrm{N}$-terminal B-type natriuretic peptide (NT-proBNP) or BNP. Blood sampling and tests including NT-proBNP were conducted by laboratories at each participating institute which were certified by The Korean Association of Quality Assurance for Clinical Laboratory. After enrollment, measurement of NT-proBNP will be performed with an electro-chemiluminescence immunoassay method using cobas ${ }^{\oplus} 8000$ (Roche Diagnostics, Mannheim, Germany) in a central laboratory. Patients will be enrolled at 13 tertiary referral centers in the Republic of Korea. Patients will be excluded if they have any of followings: low blood pressure (sitting systolic blood pressure $<90 \mathrm{mmHg}$ ), bradycardia (resting heart rate $<50$ beats $/ \mathrm{min}$ ), ischemic heart disease (unstable angina, myocardial infarction) within 1 month, severe cerebrovascular accident, respiratory diseases with bronchospasm, and peripheral vascular diseases. The detailed inclusion and exclusion criteria are listed in Table 1.

\section{Endpoints}

The primary endpoint is the change in $\mathrm{N}$-terminal pro B-natriuretic peptide (NT-proBNP) level from baseline to 6 months after randomization. The secondary endpoints include the proportion of patients with NTproBNP increment $>10 \%$ from baseline, composite of all-cause mortality and readmission, mortality rate, readmission rate, change in systolic and diastolic blood pressure at sitting position, control rate and response rate of blood pressure among patients with elevated blood pressure at baseline, quality of life assessed by the Minnesota Living with Heart Failure Questionnaire and Visual Analog Scale, and drug compliance. Table 2 demonstrates the detailed endpoints.

\section{Randomization}

Subjects who meet the inclusion and exclusion criteria will be randomly assigned to the carvedilol-SR or carvedilol-IR groups, respectively. The randomization table will be generated by an independent statistician using the SAS system's randomization program. Restricted block randomization is applied in order to ensure that the subjects are assigned to each group in a balanced manner. At this time, the size of the block will
Table 1 Inclusion and exclusion criteria

Inclusion criteria

1 Men or women aged 20 years or older

2 Confirmed left ventricular ejection fraction $\leq 40 \%$ by echocardiography within the pre-analytical 6 months

3 NT-proBNP level $\geq 125 \mathrm{pg} / \mathrm{ml}$ or BNP level $\geq 35 \mathrm{pg} / \mathrm{ml}$ within the pre-analytical 3 months

4 Clinically stable patient without evidence of congestion or extracellular fluid retention

5 Patients providing written informed consent

Exclusion criteria

1 Systolic blood pressure in a sitting position $<90 \mathrm{mmHg}$ or resting heart rate $<50$ beats/min at screening

2 Patient has a contraindication to beta-blockers

3 Patient who are expected to take another beta-blocker after randomization

4 Cardiovascular diseases:

Ischemic heart disease (unstable angina, myocardial infarction) within 1 month

Hypertrophic cardiomyopathy

Cor pulmonale

Hemodynamically significant stenosis of the aorta, aortic valve, or mitral valve

Acute myocardial infarction with complications

5 Severe cerebrovascular accident (for example, ischemic stroke or cerebral hemorrhage) within the pre-analytical within 6 months

6 Glottic edema, allergic rhinitis, respiratory diseases with bronchospasm such as asthma and chronic obstructive lung disease

7 Peripheral vascular disease (for example, Raynaud's syndrome, intermittent claudication)

8 Patients who needs vasopressor support due to prominent volume retention/overload

9 Moderate-to-severe retinopathy (for example, retinal hemorrhage, $v$ isual disturbance, retinal microaneurysm within 6 months)

10 Impaired renal function (serum creatinine $\geq 2.5 \mathrm{mg} / \mathrm{dL}$ ) or hepatic function (AST or ALT $\geq 3 \times$ ULM)

11 Patients in a clinical status that can significantly influence absorption, distribution, metabolism, and secretion of drugs for clinical trials: History of major gastrointestinal surgery, such as gastrectomy or gastric bypass surgery

Inflammatory bowel disease within 12 months

Current gastric ulcer, pancreatic function abnormality including pancreatitis, gastrointestinal/rectal bleeding which requires treatment Current urologic stenosis or obstruction which requires treatment

12 Confirmed or suspected drug/alcohol abuse within 6 months

13 Pregnant or lactating women, suspected pregnant women or lactating women

14 Chronic inflammatory diseases which require anti-inflammatory treatment

15 Hypersensitivity to carvedilol

16 Malignant disease including lymphoma and leukemia within 5 years

17 Patients who were prescribed other medication for any other clinical trials within the pre-analytical 28 days

18 Patients who are expected to have prolonged hospital stay due to other medical problems other than chronic heart failure (for example, femoral neck fracture)

19 Patients who are considered inappropriate by researchers to participate in the clinical trial

$A L T$ alanine transaminase, $A S T$ aspartate transaminase, NT-proBNP N-terminal pro B-type natriuretic peptide, ULM upper limit of meta-stability 
Table 2 Primary and secondary endpoints

\begin{tabular}{|c|c|}
\hline & Endpoint details \\
\hline $\begin{array}{l}\text { Primary } \\
\text { endpoint }\end{array}$ & $\begin{array}{l}\text { Change in of NT-proBNP level from baseline to } 6 \text { months } \\
\text { after randomization }\end{array}$ \\
\hline \multirow[t]{6}{*}{$\begin{array}{l}\text { Secondary } \\
\text { endpoints }\end{array}$} & $\begin{array}{l}\text { The frequency of NT-proBNP increment }>10 \% \text { from } \\
\text { baseline }\end{array}$ \\
\hline & Composite of all-cause mortality and readmission \\
\hline & All-cause mortality rate and readmission rate \\
\hline & $\begin{array}{l}\text { Change in systolic/diastolic blood pressure at } \\
\text { sitting position, control/response rate of } \\
\text { blood pressure }\end{array}$ \\
\hline & $\begin{array}{l}\text { Quality of life assessment by the MLHFQ, Visual } \\
\text { Analog Scale }\end{array}$ \\
\hline & Drug compliance \\
\hline
\end{tabular}

MLHFQ Minnesota Living with Heart Failure Questionnaire, NT-proBNP N-terminal pro B-type natriuretic peptide

be set by a multiple of 2 (for example, 2, 4, 68, etc.) and it will be implemented using the uniform random-number generator in SAS version 9.2. Webbased randomization will be used and all investigators will be masked to this process until the allocation has been determined.

\section{Carvedilol treatment protocol}

In this study, we will use carvedilol (trade name: Dilatrend ${ }^{\circ}$ ) which is manufactured and distributed by Chong Kun Dang Pharmaceutical Corporation in the Republic of Korea. After randomization, patients will receive either carvedilol-IR twice a day or carvedilolSR once a day for 6 months. Patients in the carvedilol-IR group will receive carvedilol-IR 3.125$\mathrm{mg}$, 6.25-mg, 12.5-mg, and 25-mg tablets twice a day, while patients in the carvedilol-SR group will receive carvedilol-SR 8-mg, 16-mg, 32-mg, and 64-mg tablets once a day. If the patients were taking beta-blockers other than carvedilol, their dose will be calculated into the equivalent dose of carvedilol (Table 3). The dose of carvedilol can be up-titrated at the discretion of the treating physician.

\section{Prohibited drugs during the clinical trial}

To avoid drug-drug interactions that may exert confounding effects, drugs with pharmacodynamic and pharmacokinetic interference will be prohibited during the clinical trial (Table 4).

\section{Clinical follow-up}

All patients will be clinically followed up at 2, 4, and 6 months after randomization at each participating center. Each clinic visit comprises history taking, physical examination, laboratory tests, and a 12-lead electrocardiograph (ECG). Drug compliance, adverse effects, and additional concomitant drugs will also be checked. Any unscheduled visit will be recorded. The detailed process is described in Table 5 .

\section{Evaluation of drug compliance}

Drug compliance will be assessed by "pill count," by comparing the number of doses remaining with the number of doses that should remain. All patients will be educated to bring the remaining tablets of carvedilol-IR or -SR to each scheduled visits during the clinical follow-up period. Examiners will count the number of returned pills and calculate the drug compliance as follows:

$$
\begin{aligned}
& \text { Drug compliance }= \\
& \frac{\text { number of pills dispensed-number of pills returned }}{\text { number of pills dispensed }}
\end{aligned}
$$

If any discrepancy is present, examiners should record the reason for the difference. Overall medication compliance should be at least $80 \%$ or more during the trial. Subjects who do not satisfy this criterion will be excluded from the per-protocol (PP) analysis.

\section{Statistical analysis \\ Sample size calculation}

The aim of the study is to evaluate whether the clinical efficacy of carvedilol-SR is non-inferior to carvedilol-IR in terms of NT-proBNP reduction in stable Asian HFrEF patients. Therefore, the sample size was calculated based on the primary endpoint, i.e., the change in NT-proBNP level from baseline to 6 months. Olsson et al. [18] showed a decrease in NT-proBNP level by $334.5 \pm$ $311.3 \mathrm{pg} / \mathrm{ml}$ in patients with chronic HF and receiving carvedilol. We established a non-inferiority margin of $110 \mathrm{pg} / \mathrm{ml}$, which corresponds to $33.3 \%$ of the mean change. With an estimated dropout rate of $10 \%$, a total of 320 patients (160 patients in each group) would be necessary to provide a power of $85 \%$ with a one-sided alpha of $2.5 \%$. This will provide $95 \%$ power to demonstrate the non-inferiority of carvedilol-SR once a day compared to carvedilol-IR twice a day (Fig. 2).

\section{Statistical analysis}

Data will be presented as numbers and frequencies for categorical variables, and as means \pm standard deviations or median with interquartile range for continuous variables. For comparisons between the carvedilol-IR group and carvedilol-SR group, the $\chi^{2}$ test or Fisher's exact test will be used for categorical variables, and the unpaired Student $t$ test will be used for continuous variables, as appropriate. Fisher's exact test will be used when the expected frequencies are less than 5 . The change in NTproBNP level from baseline to 6 months after randomization will be analyzed with the paired $t$ test. In addition, analysis of covariance (ANCOVA) will be also 
Table 3 Equivalent dose of carvedilol with other beta-blockers

\begin{tabular}{lllll}
\hline & Dose & & & \\
\hline Carvedilol IR & $3.125 \mathrm{mg} \mathrm{bid}$ & $6.25 \mathrm{mg} \mathrm{bid}$ & $12.5 \mathrm{mg} \mathrm{bid}$ & $25 \mathrm{mg} \mathrm{bid}$ \\
Carvedilol SR & $8 \mathrm{mg} \mathrm{qd}$ & $16 \mathrm{mg} \mathrm{bid}$ & $32 \mathrm{mg} \mathrm{bid}$ & $64 \mathrm{mg} \mathrm{qd}$ \\
Bisoprolol & $1.25 \mathrm{mg} \mathrm{qd}$ & $2.5 \mathrm{mg} \mathrm{qd}$ & $5 \mathrm{mg} \mathrm{qd}$ & $10 \mathrm{mg} \mathrm{qd}$ \\
Nebivolol & $1.25 \mathrm{mg} \mathrm{qd}$ & $2.5 \mathrm{mg} \mathrm{qd}$ & $5 \mathrm{mg} \mathrm{qd}$ & $10 \mathrm{mg} \mathrm{qd}$ \\
Metoprolol succinate & $25 \mathrm{mg} \mathrm{qd}$ & $50 \mathrm{mg} \mathrm{qd}$ & $100 \mathrm{mg} \mathrm{qd}$ & $200 \mathrm{mg} \mathrm{qd}$ \\
\hline
\end{tabular}

bid twice daily, IR immediate-release, ad four times daily, $S R$ slow-release

conducted to analyze the change of NT-proBNP. The outcomes of chronological trend, such as mortality and readmission rate, will be assessed using KaplanMeier estimates. The log-rank test will be used to compare differences in clinical outcomes between the groups. A multivariable Cox proportional hazard regression model will be used to adjust for significant covariates. The data will be primarily analyzed according to the intention-to-treat rule including all randomized subjects. We also plan a PP analysis for patients with drug compliance of $80 \%$ or less. Onesided $p$ values $<0.05$ will be considered statistically significant. The analyses will be performed by a professional statistician.

\section{Trial organization}

\section{Executive Committee}

The Executive Committee will be composed of the study chairperson and the principal investigators of the investigating centers. This committee will approve the final trial design and protocol issued to the Data and Safety Monitoring Board (DSMB) and the clinical sites. This committee will also be responsible for reviewing the final results, determining the methods of presentation and publication, and the selection of secondary projects and publications by members of the Steering Committee.

\section{Data Safety Monitoring Board (DSMB)}

An independent DSMB will be composed of cardiologists and a biostatistician, all of whom did not participate in the trial, following the applicable regulatory guidelines. The DSMB will review the safety data from this study and will make recommendations based on safety analyses of unanticipated device effects (UADEs), serious adverse events (SAEs), protocol deviations, and follow-up reports. In addition to the scheduled DSMB meetings (which will be determined prior to the initiation of the study) the board will convene a meeting at any time if safety problems become an issue. The DSMB is responsible for recommending to the Executive Committee to modify or stop the study if there are any safety or compliance issues. However, the final decision regarding study modifications will rest with the Executive Committee. Cumulative safety data will be reported to the DSMB and will be reviewed on an ongoing basis throughout enrollment and follow-up to ensure patient safety. Every effort will be made to allow the DSMB to conduct an unbiased review of the patients' safety information. All DSMB reports will be made available to the appropriate agencies upon request but otherwise will remain strictly confidential. Prior to the DSMB's first review of the data, the DSMB charter will be drafted. This plan defines the stopping rules

Table 4 Contraindicated drugs during clinical trial

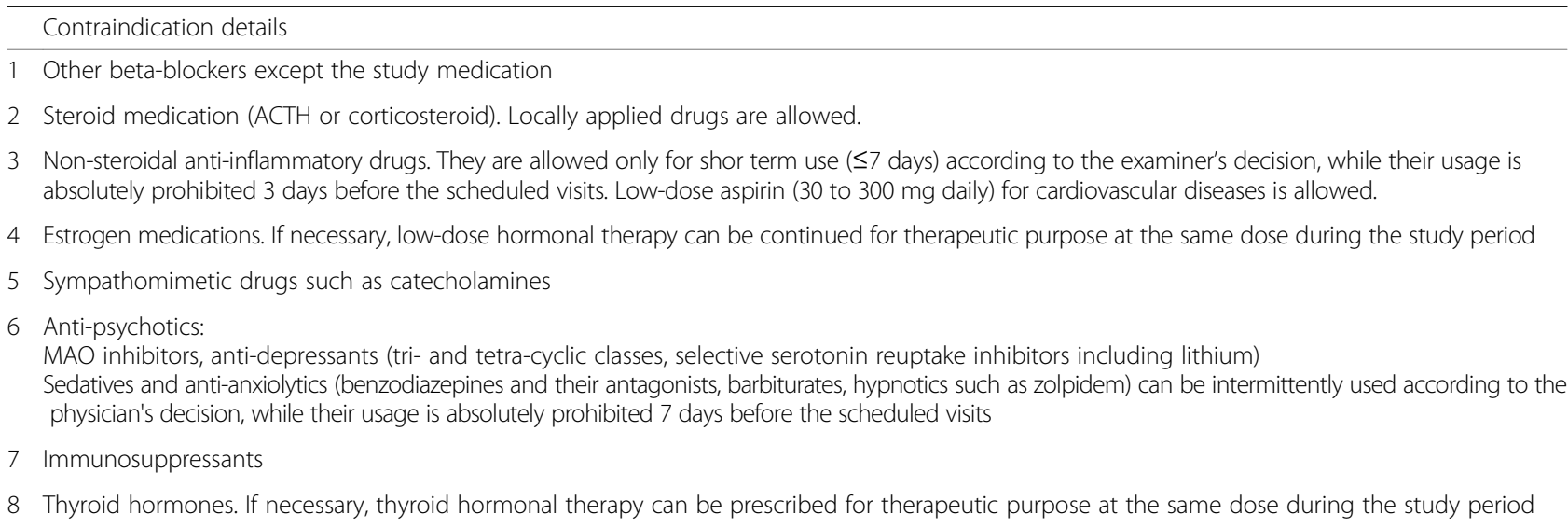


Table 5 Trial process chart

\begin{tabular}{|c|c|c|c|c|c|}
\hline Visit & Visit 1 & Visit 2 & Visit 3 & Visit 4 & Visit 5 \\
\hline $\begin{array}{l}\text { Status } \\
\text { Period } \\
\end{array}$ & $\begin{array}{l}\text { Screening } \\
-2 \text { to } 0 \text { week }\end{array}$ & $\begin{array}{l}\text { Baseline } \\
0 \text { months }\end{array}$ & $\begin{array}{l}2 \text { months } \\
( \pm 4 \text { days })\end{array}$ & $\begin{array}{l}4 \text { months } \\
( \pm 14 \text { days) }\end{array}$ & $\begin{array}{l}6 \text { months } \\
( \pm 14 \text { days })\end{array}$ \\
\hline Inclusion/exclusion criteria & $\cdot$ & & & & \\
\hline Informed consent & - & & & & \\
\hline Demographic data & $\cdot$ & & & & \\
\hline Past medical history ${ }^{a}$ & . & & & & \\
\hline Randomization & & $\cdot$ & & & \\
\hline Physical examination & $\cdot$ & $\cdot$ & $\cdot$ & - & $\cdot$ \\
\hline Vital signs & $\cdot$ & $\cdot$ & $\cdot$ & $\cdot$ & $\cdot$ \\
\hline Height and weight ${ }^{b}$ & . & . & . & . & · \\
\hline Blood analysis ${ }^{c}$ & - & . & & & - \\
\hline Urinalysis $^{d}$ & . & . & & & . \\
\hline Pregnancy test $\mathrm{e}^{\mathrm{e}}$ & . & . & & & $\cdot$ \\
\hline Chest $x$-ray ${ }^{f}$ & . & & & & \\
\hline 12-lead electrocardiograph ${ }^{9}$ & - & & & & - \\
\hline Echocardiography ${ }^{\text {h }}$ & . & - & & & · \\
\hline Concomitant medication & $\cdot$ & $\cdot$ & $\cdot$ & $\cdot$ & $\cdot$ \\
\hline Adverse events & & $\cdot$ & $\cdot$ & $\cdot$ & $\cdot$ \\
\hline Patient's compliance & & & $\cdot$ & $\cdot$ & $\cdot$ \\
\hline Questionnaire for quality of life & & $\cdot$ & & & - \\
\hline
\end{tabular}

${ }^{a}$ The history of diseases, medications, and operations will be recorded

${ }^{\mathrm{b}}$ Check weight only after visit 2

${ }^{C} \mathrm{CBC}$ : WBC with differential count, hemoglobin, hematocrit, platelets; chemistry: albumin, total protein, triglycerides, high-density lipoprotein-cholesterol, low-density lipoprotein-cholesterol, blood urea nitrogen, serum creatinine, uric acid, total bilirubin, AST, ALT, ALP, NT-proBNP, hs-CRP; electrolytes: Na, K, Ca, Cl, P

${ }^{\mathrm{d}}$ Specific gravity, pH, albumin (protein), glucose, blood (occult blood), urobilinogen, ketones

eWomen of child-bearing age

f,g Examination at visit 6 can be optionally performed according to the examiner's decision

${ }^{h}$ Follow-up echocardiograph, if available

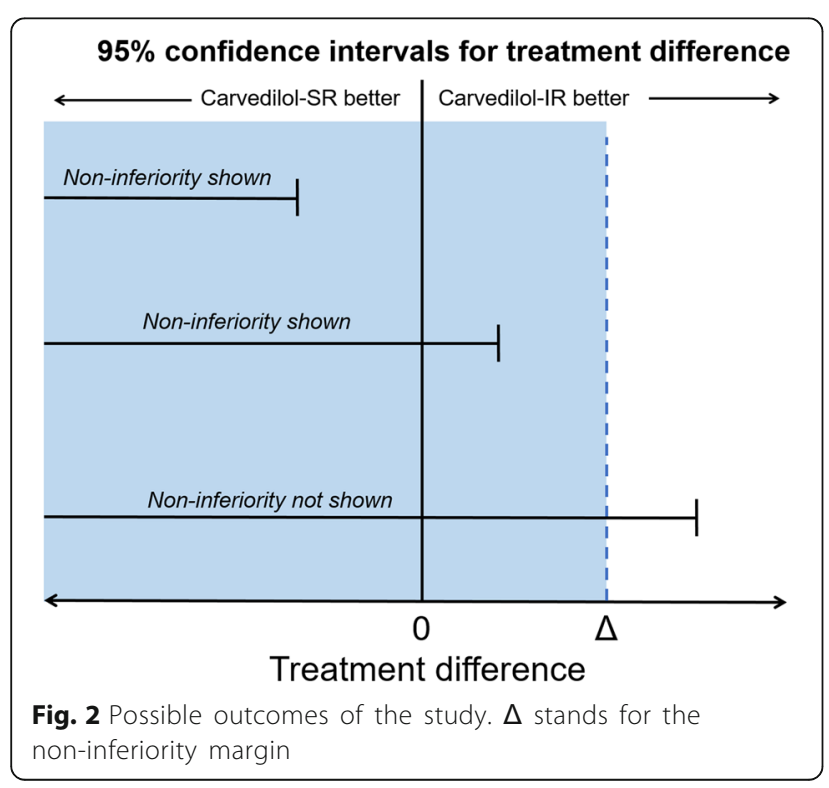

for stopping the trial for safety. The first meeting of the DSMB will be requested for discussion of the protocol and an understanding of all the protocol elements. The DSMB will develop a consensus understanding of all trial endpoints and definitions used in the event adjudication process.

\section{Clinical Event Adjudication Committee (CEAC)}

The Clinical Events Adjudication Committee (CEAC) consists of cardiologists who do not participate in this study. The CEAC has responsibility to develop specific criteria used for the categorization of clinical events and clinical endpoints in the study, which are based on protocol. At the onset of the trial, the CEAC establishes regulations mentioning the minimum amount of data required and the algorithm followed in order to classify a clinical event. All members of the CEAC are blinded to the primary results of the study and meet regularly to review and adjudicate all clinical events in which the required minimum data is available. The CEAC also reviews and rules on all deaths that may occur during the trial. 


\section{Data coordination and site management}

Data coordination and site management services are performed by the Clinical Trials Center at Seoul National University Bundang Hospital.

\section{Ethical approval}

This clinical trial was approved by the Institutional Review Board or Ethics Committee at each of the 13 participating hospitals; they are: Seoul National University Bundang Hospital, Seoul National University Hospital, Yonsei University Severance Hospital, Wonju Severance Christian Hospital, Samsung Medical Center, Sejong General Hospital, Ajou University Hospital, Boramae Medical Center, Hallym University Medical Center, Korea University Guro Hospital, Korea University Anam Hospital, Seoul Medical Center, and Asan Medical Center. The investigation will conform to the principles outlined in the Declaration of Helsinki. The study protocol has been registered at ClinicalTrials.gov (NCT03209180). All authors are responsible for the study design, data acquisition, data analysis, manuscript writing and editing.

\section{Discussion}

After an initial cardiac injury, there is an increase in neuro-humoral activity, i.e., the sympathetic nervous system and renin-angiotensin-aldosterone system [5]. This response can temporarily increase the myocardial contractility [19]; however, sustained over-activation can lead to the development and progression of HF [20]. Thus, angiotensin-converting enzyme inhibitors/angiotensin-receptor blockers and beta-blockers have been used to attenuate the neuro-humoral over-activation and have been shown to improve the prognosis of patients with HFrEF. Unlike to renin-angiotensin system blockers (RAS blockers), only four beta-blockers, i.e., carvedilol, metoprolol succinate, bisoprolol, and nebivolol, have demonstrated a beneficial effect. For example, bucindolol and propranolol failed to show any clinical benefit, suggesting that the beneficiary effect of beta-blockers is not "a class effect" but a substrate-specific effect [6, 7, 21-23]. Therefore, the current clinical practice guidelines for managing HF patients provide detailed information on the drug name and target dose for each agent $[1,11]$.

Carvedilol has been extensively evaluated in HFrEF patients with mild-to-severe LV dysfunction $[8,9,24]$ and has demonstrated remarkable efficacy in improving clinical outcomes [10]. Unlike bisoprolol and metoprolol succinate, carvedilol has a short elimination half-life, so that it needs to be administrated twice a day [12]. Many HF patients have advanced age and have multiple comorbidities, so that they take multiple drugs $[2,25]$. A complex medication regimen causes reduced drug compliance and, consequently, can lead to worse clinical outcomes $[14,16,26]$. In addition, the high drug adherence observed in well- controlled clinical trials may differ from that in realworld clinical practice. To increase the drug compliance of carvedilol-IR, longer-acting carvedilol-SR has been developed.

Carvedilol-SR meets the basic criteria for a controlled release agent: (1) it maintains an effective plasma concentration throughout the day and (2) does not exceed the critical value above which side effects often develop [27]. In addition, regarding the pharmacokinetic profile, carvedilol-SR once daily was similar to that for carvedilol-IR given twice daily [27].

The SLOW-HF study is a randomized, open-label. phase-IV clinical trial that investigates the clinical benefit and safety of carvedilol-SR compared to carvedilol-IR in patients with HFrEF. If carvedilol-SR is not inferior to carvedilol-IR in terms of clinical efficacy and safety, it would be a useful therapeutic option to improve medication complexity and compliance in managing HFrEF patients.

\section{Study limitations}

There are several limitations. First, the primary endpoint of our study is the change in NT-proBNP level from baseline to the study end. Although NT-proBNP is a well-validated marker for risk stratification and used as a surrogate marker for clinical outcomes, clinical endpoints, such as mortality and rehospitalization for worsening HF, would be more definitive. Second, there exists a possibility that patients with more advanced HF may be under-represented in this study because they often have fluid volume retention. However, similar findings have been observed in other clinical trials which investigated the effect of beta-blocker management in HF patients $[6,7,10,21]$. Finally, this is an open-label study.

\section{Trial status}

The trial is currently in the recruitment phase (recruitment began on 27 October 2016 and is expected to finish on 31 December 2018).

\section{Additional file}

Additional file 1: SPIRIT 2013 Checklist. (DOC 124 kb)

\section{Abbreviations}

CEAC: Clinical Event Adjudication Committee; DSMB: Data and Safety Monitoring Board; HF: Heart failure; HFrEF: Heart failure with reduced ejection fraction; IR: Immediate release; LVEF: Left ventricular ejection fraction; NT-proBNP: N-terminal pro B-natriuretic peptide; PP: Per-protocol; SR: Slow release

\section{Acknowledgements}

None.

\section{Funding}

This project is an investigator-initiated trial and is funded by an unrestricted grant from Chong Kun Dang Pharmaceutical Corporation. Chong Kun Dang 
is a manufacturer of the original formulation of carvedilol in the Republic of Korea. The funding source has no involvement in the current study.

\section{Availability of data and materials}

Data sharing is not yet applicable to this article as no datasets were generated or analyzed during the current study.

\section{Authors' contributions}

D-JC, CSP, and JJP contributed to the conception and design of the this study, and wrote the draft of the study protocol. H-YL, S-MK, B-SY, E-SJ, SKH, J-HS, M-AK, D-GP, E-JK, S-JH, S-YK, and J-JK took part in the coordination of the trial. All authors read and provided final approval of the manuscript submitted.

\section{Ethics approval and consent to participate}

This clinical trial was approved by the Institutional Review Board or Ethics Committee at each of the 13 participating hospitals. Informed consent is made before registration.

\section{Consent for publication}

Not applicable

\section{Competing interests}

The authors declare that they have no competing interests.

\section{Publisher's Note}

Springer Nature remains neutral with regard to jurisdictional claims in published maps and institutional affiliations.

\section{Author details \\ ${ }^{1}$ Department of Internal Medicine, Seoul National University College of Medicine, Seoul National University Bundang Hospital, Seongnam, South Korea. ${ }^{2}$ Department of Internal Medicine, Seoul National University College of Medicine, Seoul National University Hospital, Seoul, South Korea. ${ }^{3}$ Division of Cardiology, Yonsei University Severance Hospital, Seoul, South Korea. ${ }^{4}$ Division of Cardiology, Yonsei University Wonju Severance Christian Hospital, Wonju, South Korea. ${ }^{5}$ Department of Internal Medicine, Sungkyunkwan University College of Medicine, Samsung Medical Center, Seoul, South Korea. ${ }^{6}$ Division or Cardiology, Sejong General Hospital, Bucheon, Gyeonggi-do, South Korea. ${ }^{7}$ Division of Cardiology, Ajou University Hospital, Suwon, Gyeonggi-do, South Korea. ${ }^{8}$ Department of Internal Medicine, Seoul National University College of Medicine, Seoul National University Boramae Medical Center, Seoul, South Korea. ${ }^{9}$ Cardiovascular Center, Hallym University Medical Center, Seoul, South Korea. ${ }^{10}$ Division of Cardiology, Korea University Guro Hospital, Seoul, South Korea. ${ }^{11}$ Division of Cardiology, Korea University Anam Hospital, Seoul, South Korea. \\ ${ }^{12}$ Department of Internal Medicine, Seoul Medical center, Seoul, South Korea. ${ }^{13}$ Department of Internal Medicine, University of Ulsan College of Medicine, Asan Medical Center, Seoul, South Korea. ${ }^{14}$ Cardiovascular Center and Division of Cardiology, Department of Internal Medicine, Seoul National University Bundang Hospital, 82 Gumi-ro 173 Beon-gil, Bundang-gu, Gyeonggi-do 13620, South Korea.}

\section{Received: 20 September 2017 Accepted: 13 January 2018}

\section{Published online: 13 February 2018}

\section{References}

1. Ponikowski P, Voors AA, Anker SD, Bueno H, Cleland JG, Coats AJ, et al. 2016 ESC Guidelines for the diagnosis and treatment of acute and chronic heart failure: The Task Force for the diagnosis and treatment of acute and chronic heart failure of the European Society of Cardiology (ESC). Developed with the special contribution of the Heart Failure Association (HFA) of the ESC. Eur Heart J. 2016;37:2129-200.

2. Benjamin EJ, Blaha MJ, Chiuve SE, Cushman M, Das SR, Deo R, et al. Heart disease and stroke statistics - 2017 update: a report from the American Heart Association. Circulation. 2017;135:e146-603.

3. Lee JH, Lim NK, Cho MC, Park HY. Epidemiology of heart failure in Korea: present and future. Korean Circ J. 2016;46:658-64.

4. Merlo M, Pivetta A, Pinamonti B, Stolfo D, Zecchin M, Barbati G, et al. Longterm prognostic impact of therapeutic strategies in patients with idiopathic dilated cardiomyopathy: changing mortality over the last 30 years. Eur J Heart Fail. 2014;16:317-24.
5. Mann DL. Mechanisms and models in heart failure: a combinatorial approach. Circulation. 1999:100:999-1008.

6. Lechat P, Brunhuber KW, Hofmann R, Kuhn P, Nesser HJ, Slany J, et al. The Cardiac Insufficiency Bisoprolol Study II (CIBIS-II): a randomised trial. Lancet. 1999;353:9-13

7. Hjalmarson A, Goldstein S, Fagerberg B, Wedel H, Waagstein F, Kjekshus J, et al. Effects of controlled-release metoprolol on total mortality, hospitalizations, and well-being in patients with heart failure: the Metoprolol CR/XL Randomized Intervention Trial in congestive heart failure (MERIT-HF). MERIT-HF Study Group. JAMA. 2000;283:1295-302.

8. Packer M, Bristow MR, Cohn JN, Colucci WS, Fowler MB, Gilbert EM, et al. The effect of carvedilol on morbidity and mortality in patients with chronic heart failure. U.S. Carvedilol Heart Failure Study Group. N Engl J Med. 1996;334:1349-55.

9. Packer M, Fowler MB, Roecker EB, Coats AJ, Katus HA, Krum H, et al. Effect of carvedilol on the morbidity of patients with severe chronic heart failure: results of the carvedilol prospective randomized cumulative survival (COPERNICUS) study. Circulation. 2002:106:2194-9.

10. Poole-Wilson PA, Swedberg K, Cleland JG, Di Lenarda A, Hanrath P, Komajda $\mathrm{M}$, et al. Comparison of carvedilol and metoprolol on clinical outcomes in patients with chronic heart failure in the Carvedilol Or Metoprolol European Trial (COMET): randomised controlled trial. Lancet. 2003:362:7-13.

11. Yancy CW, Jessup M, Bozkurt B, Butler J, Casey Jr DE, Drazner MH, et al. 2013 ACCF/AHA guideline for the management of heart failure: executive summary: a report of the American College of Cardiology Foundation/ American Heart Association Task Force on practice guidelines. Circulation. 2013:128:1810-52

12. Keating GM, Jarvis B. Carvedilol: a review of its use in chronic heart failure. Drugs. 2003;63:1697-741

13. Udelson JE, Pressler SJ, Sackner-Bernstein J, Massaro J, Ordronneau P, Lukas $M A$, et al. Adherence with once daily versus twice daily carvedilol in patients with heart failure: the Compliance and Quality of Life Study Comparing Once-Daily Controlled-Release Carvedilol CR and Twice-Daily Immediate-Release Carvedilol IR in Patients with Heart Failure (CASPER) Trial. J Card Fail. 2009:15:385-93.

14. Mansur N, Weiss A, Beloosesky Y. Looking beyond polypharmacy: quantification of medication regimen complexity in the elderly. Am J Geriatr Pharmacother. 2012;10:223-9.

15. Toh MR, Teo V, Kwan YH, Raaj S, Tan SY, Tan JZ. Association between number of doses per day, number of medications and patient's noncompliance, and frequency of readmissions in a multi-ethnic Asian population. Prev Med Rep. 2014;1:43-7.

16. Colavecchia AC, Putney DR, Johnson ML, Aparasu RR. Discharge medication complexity and 30-day heart failure readmissions. Res Social Adm Pharm. 2017:13:857-63.

17. Lang RM, Badano LP, Mor-Avi V, Afilalo J, Armstrong A, Ernande L, et al. Recommendations for cardiac chamber quantification by echocardiography in adults: an update from the American Society of Echocardiography and the European Association of Cardiovascular Imaging. J Am Soc Echocardiogr. 2015;28:1-39. e14.

18. Olsson LG, Swedberg K, Cleland JG, Spark PA, Komajda M, Metra M, et al. Prognostic importance of plasma NT-pro BNP in chronic heart failure in patients treated with a beta-blocker: results from the Carvedilol Or Metoprolol European Trial (COMET) trial. Eur J Heart Fail. 2007:9:795-801.

19. Baker AJ. Adrenergic signaling in heart failure: a balance of toxic and protective effects. Pflugers Arch. 2014;466:1139-50.

20. Rozanski A, Blumenthal JA, Kaplan J. Impact of psychological factors on the pathogenesis of cardiovascular disease and implications for therapy. Circulation. 1999.99.2192-217.

21. Flather MD, Shibata MC, Coats AJ, Van Veldhuisen DJ, Parkhomenko A, Borbola J, et al. Randomized trial to determine the effect of nebivolol on mortality and cardiovascular hospital admission in elderly patients with heart failure (SENIORS). Eur Heart J. 2005:26:215-25.

22. Cleland JG, Bristow MR, Erdmann E, Remme WJ, Swedberg K, Waagstein F. Beta-blocking agents in heart failure. Should they be used and how? Eur Heart J. 1996;17:1629-39.

23. Eichhorn EJ, Domanski MJ, Krause-Steinrauf H, Bristow MR, Lavori PW. A trial of the beta-blocker bucindolol in patients with advanced chronic heart failure. N Engl J Med. 2001:344:1659-67.

24. Dargie HJ. Effect of carvedilol on outcome after myocardial infarction in patients with left-ventricular dysfunction: the CAPRICORN randomised trial. Lancet. 2001;357:1385-90. 
25. Baron-Franco B, McLean G, Mair FS, Roger VL, Guthrie B, Mercer SW. Comorbidity and polypharmacy in chronic heart failure: a large crosssectional study in primary care. Br J Gen Pract. 2017;67:e314-20.

26. Yam FK, Lew T, Eraly SA, Lin HW, Hirsch JD, Devor M. Changes in medication regimen complexity and the risk for 90-day hospital readmission and/or emergency department visits in U.S. Veterans with heart failure. Res Social Adm Pharm. 2016;12:713-21.

27. Packer M, Lukas MA, Tenero DM, Baidoo CA, Greenberg BH, Study G. Pharmacokinetic profile of controlled-release carvedilol in patients with left ventricular dysfunction associated with chronic heart failure or after myocardial infarction. Am J Cardiol. 2006;98:39-45.

Submit your next manuscript to BioMed Central and we will help you at every step:

- We accept pre-submission inquiries

- Our selector tool helps you to find the most relevant journal

- We provide round the clock customer support

- Convenient online submission

- Thorough peer review

- Inclusion in PubMed and all major indexing services

- Maximum visibility for your research

Submit your manuscript at www.biomedcentral.com/submit
Biomed Central 